\title{
Supramolecular Approach to Decorate Multi-Walled Carbon Nanotubes with Negatively Charged Iron(II) Complexes
}

\author{
Sergio A. V. Jannuzzi, ${ }^{a, b}$ Bianca Martins, ${ }^{a}$ Luis E. S. C. Huamani ${ }^{a}$ and André L. B. Formiga ${ }^{*, a}$ \\ ${ }^{a}$ Instituto de Química, Universidade Estadual de Campinas, UNICAMP, CP 6154, 13083-970 Campinas-SP, Brazil \\ ${ }^{b}$ Department of Chemistry, KU Leuven, Celestijnenlaan 200F, B-3001 Leuven, Belgium
}

\begin{abstract}
Poly(4-vinylpyridine) (P4VP) and P4VP/pentacyanoferrate(II) metallopolymer were used to suspend up to $1 \mathrm{mg} \mathrm{mL}^{-1}$ of multi-walled carbon nanotubes (MWCNT) in ethanol/water mixtures, providing noncolavent decoration of MWCNT with electroactive iron complex. The major interaction between polymer side chains and nanotubes is via $\pi$ - $\pi$ stacking, rather than chargetransfer interaction reported for many nitrogenated interacting molecules. Preservation of structural integrity of the nanotubes after functionalization was attested by Raman, which was reflected in the semiconducting character of the dried nanocomposites. Proximity of pentacyanoferrate(II) to MWCNT walls was suggested by its solvatochromic shift of metal-to-ligand charge transfer band attributed to $\left[\mathrm{Fe}(\mathrm{CN})_{5}\right]^{3-}$ bound to P4VP pyridyl moieties. Electronic microscopy revealed smooth films and good adhesion between MWCNT bundles and the matrix. The procedure described herein can be used to combine unique properties of transition metal compounds able to coordinate to pyridyl groups, with the film-forming ability of P4VP and conductivity provided by MWCNT.
\end{abstract}

Keywords: metallopolymer, carbon nanotube decoration, pentacyanoferrate

\section{Introduction}

Carbon nanotubes (CNTs) are one of the most studied objects in the field of materials science due to their interesting electrical, thermal and mechanical properties. ${ }^{1}$ Decoration of CNTs with coordination compounds can be achieved by covalent bonding using different reactions, leading to functionalization with, e.g., porphyrins, ${ }^{2,3}$ phthalocyanines, ${ }^{4,5}$ bipyridine, ${ }^{6}$ terpyridine, ${ }^{7,8}$ and catechol ${ }^{9}$ complexes.

Chemical modification of CNT surface is frequently performed by fluorination or by strong oxidizing agents, so that polar functional groups can be created to allow dispersion in solvents and subsequent bond formation with anchoring groups. ${ }^{10-12}$ This method usually creates defects in the CNT lattice, lowering its electrical and thermal conductivity and compromising its mechanical properties. ${ }^{13}$ To overcome this drawback, some groups explored noncovalent functionalization through van der Waals forces with pyrene derivatives, ${ }^{14-16}$ surfactants, ${ }^{17}$ and also with standard Werner-type complexes. ${ }^{18}$ After functionalization (of both kinds), the material can be

*e-mail: formiga@iqm.unicamp.br processed as a dispersion in some common solvent, which allows its application for different purposes, as production of printed electrodes ${ }^{19}$ and phenolic resin nanocomposite, ${ }^{17}$ for example.

The association of CNTs with polymers provides films with enhanced electrical conductivity and superior mechanical performance provided that hydrophobic polymer chains can overcome van der Waals interactions that sustain CNTs together in bundles. ${ }^{20-24}$ Wrapping molecules can bind onto CNT outermost surface through other non-covalent interaction like acid-base adduct formation, also known as charge-transfer interaction. ${ }^{13}$ This kind of strategy has been used to build very stable decorated CNTs with different transition metal compounds. ${ }^{25-27}$

The affinity of semiconductor single-walled carbon nanotubes (SWCNT) towards basic nitrogen atoms is well-known. ${ }^{28-30}$ As reported by Rouse, ${ }^{31}$ poly(4vinylpyridine) (P4VP) performs well the role of bridge between CNT and solvent and hence is able to disperse them in alcoholic medium. P4VP was used by Vos and co-workers $^{32}$ to obtain stable suspensions of SWCNT or multi-walled carbon nanotube (MWCNT) with P4VPbased metallopolymers containing $\left[\mathrm{M}(\mathrm{bpy})_{2} \mathrm{Cl}\right] \mathrm{Cl}$, where $\mathrm{M}$ is $\mathrm{Ru}^{2+}$ or $\mathrm{Os}^{2+}$ and bpy is 2,2'-bipyridine. They show 
that the electronic and electrochemical properties of redox metallopolymers do not change after addition of CNT, thus letting to CNT the role of improving the mechanical properties of the material. Furthermore, they suggest that the suspension-forming ability is due to the interaction of CNT walls with metallopolymer ligands via $\pi-\pi$ stacking. In another work, ${ }^{33}$ a biosensor for glucose was produced from pyridyl-containing chitosan complexed with pentacyanoferrate. The polymeric matrix embedded glucose oxidase while pentacyanoferrate served as electrochemical mediator. Differently from Vos and co-workers, ${ }^{32}$ addition of MWCNT improved the overall electrochemical response but the specific interaction of the redox mediator with the CNTs was not investigated.

The association of P4VP, MWCNT and pentacyanoferrate(II) has been shown previously by us to be a good electrocatalyst for cysteine oxidation at low overpotential. ${ }^{34}$ Despite the successful application of the hybrid material in electrochemical sensing, the interaction between the components lacks investigation. It is still not clear whether acid-base interaction between the nitrogen atom of P4VP and the outermost wall of CNTs is predominant over $\pi-\pi$ interactions. We report herein the characterization of this solution-processable (the term "solution" here is taken loosely to refer indeed to a "suspension" in order to express the idea that the material can be handled as easy as any solution) multifunctional material, which gathers (i) electrical conductivity of CNT; (ii) film-forming ability of P4VP and (iii) its solvation properties that grants MWCNT dispersion, and (iv) demonstrated electrochemical activity of the complex. Potentially, properties of any other complex with a labile ligand able to be substituted by the pendant pyridyl moieties of the polymer could be further integrated to those of the other two components. Moreover, the simple procedure reported herein can be employed in the production of new P4VP-based blends, thin semiconducting films as well as in the design of functional solution-processable metal-containing architectures based on P4VP copolymers.

\section{Experimental}

\section{Instrumentation}

UV-Vis spectra were recorded on a diode array Agilent spectrophotometer model HP 8453. The ultrasonication bath used was a Thornton Chubby, $240 \mathrm{~W}, 40 \mathrm{kHz}$ from Inpec Eletrônica. Centrifugation was carried out in a Quimis bench centrifuge. Raman spectroscopy was carried out using a Horiba Jobin-Yvon T64000 triple spectrometer system, equipped with a confocal microscope and a nitrogen-cooled charge coupled device (CCD) detector. The spectrum was obtained with the $514.5 \mathrm{~nm}$ line of a $\mathrm{Ar} / \mathrm{Kr}$ laser $(7.4 \mathrm{~mW})$ and accumulation time of $180 \mathrm{~s}$ at room temperature. The samples were drop-cast onto a microscope glass slide and they were let to dry at room temperature. The spectra were acquired from the center of the drop-cast film, because they were less subjected to variation of the relative intensity of the bands than spectra acquired in regions closer to the edges of the film. Microscopy investigations were carried out in a JEOL JSM 6360 LV scanning electron microscope operating at $20 \mathrm{kV} .{ }^{1} \mathrm{H}$ and ${ }^{13} \mathrm{C}$ nuclear magnetic resonance (NMR) spectra were recorded on a Bruker Avance $500 \mathrm{MHz}$ spectrometer operating at 499.87 and $125.69 \mathrm{MHz}$, respectively. ${ }^{1} \mathrm{H}-{ }^{15} \mathrm{~N}$ data were acquired on a Bruker Avance $500 \mathrm{MHz} .{ }^{15} \mathrm{~N}$ NMR chemical shift were indirectly detected in solution by $\left[{ }^{1} \mathrm{H}-{ }^{15} \mathrm{~N}\right]$ heteronuclear multiple bond coherence (HMBC) experiment. The signals were calibrated using the signal at $1.11 \mathrm{ppm}$ of residual ethanol. All samples were prepared in ethanol- $d_{6}$. The DC electrical conductivity of the films was measured using a four-point probe (Cascade Microtech C4S-64) coupled with an electrometer (Keithley 617) and a digital multimeter (Minipa ET-2500).

\section{Materials}

Poly(4-vinylpyridine) (P4VP, 99\%, Mw $=160.000$, Aldrich); multi-walled carbon nanotubes (95\%, MWCNTs Co., Ltd.), external diameter: 10-40 nm, length: 5-20 $\mu \mathrm{m}$; absolute ethanol (Merck); ethanol- $d_{6}$ (D 99\%, anhydrous, Cambridge Isotopes Laboratories, Inc.); hydrochloric acid (36.5-38\%, Baker Analyzed); sodium nitroprusside $\left(\mathrm{Na}_{2}\left[\mathrm{Fe}(\mathrm{CN})_{5} \mathrm{NO}\right] .2 \mathrm{H}_{2} \mathrm{O}, 99 \%\right.$, Merck); ammonium hydroxide (Merck) were used as received without further purification.

\section{Synthesis of $\mathrm{Na}_{3}\left[\mathrm{Fe}(\mathrm{CN})_{5} \mathrm{NH}_{3}\right] \cdot 3 \mathrm{H}_{2} \mathrm{O}$}

The procedure for synthesis of sodium aminopentacyanoferrate used sodium nitroprusside in concentrated ammonium hydroxide, as described in the literature. ${ }^{35}$

\section{Synthesis of P4VP-Fe(CN) $)_{5}$ metallopolymer}

Two metallopolymer samples were prepared, as described elsewhere, ${ }^{36}$ having 50:1 and 200:1 mol of polymer repeating units per mol of $\left[\mathrm{Fe}(\mathrm{CN})_{5}\right]^{3-}$, henceforth named as py:Fe ratio. Therefore the first sample has four times more pentacyanoferrate than the second. 


\section{Formation of MWCNT suspensions}

Mixtures of MWCNTs, P4VP (or P4VP-Fe(CN) metallopolymer) and ethanol with different proportion of these components were prepared, as presented in Table 1. A $42.2 \mathrm{mg} \mathrm{mL}^{-1} \mathrm{P} 4 \mathrm{VP}$ solution in ethanol and freshly-prepared solutions of the metallopolymers were used to prepare the samples with pure P4VP and with the metallopolymers, respectively. In the case of samples with metallopolymers, two values of P4VP/MWCNT weight ratio were tested. The necessary volume to obtain the desired mass of P4VP in the mixture was transferred to a test tube to which was added ethanol up to the final volume of $3.5 \mathrm{~mL}$. Then, the amount of MWCNTs was added and the mixture was sonicated for 2 hours uninterruptedly.

\section{Quantification of MWCNT in the suspensions}

To $1.0 \mathrm{~mL}$ of the suspension containing P4VP or P4VP-Fe(CN) 5 metallopolymer was added $4 \mathrm{~mL}$ of concentrated $\mathrm{HCl}$. The flocculated MWCNT was separated by centrifugation, washed with $4 \mathrm{~mL}$ of $\mathrm{HCl}$ $1.0 \mathrm{~mol} \mathrm{~L}^{-1}$ and centrifuged again. The UV-Vis spectra of the supernatant was acquired. The presence of P4VP and the respective protonated species were attested by the characteristic band of the aromatic ring at $257 \mathrm{~nm}$. The supernatant was discarded and a new portion of $4 \mathrm{~mL}$ of $\mathrm{HCl} 1.0 \mathrm{~mol} \mathrm{~L}^{-1}$ was added. Several cycles of washing and centrifugation were performed until the UV band vanishes, indicating that the recovered MWCNT is free from the polymer. The cyclic procedure is shown in Figure 1. Subsequent washing steps (approximately 12) were done with $4 \mathrm{~mL}$ of deionized water until rinsed water $\mathrm{pH}$ becomes neutral. After that, the supernatant was removed, the MWCNT free from P4VP and acid was vacuum dried and weighted.

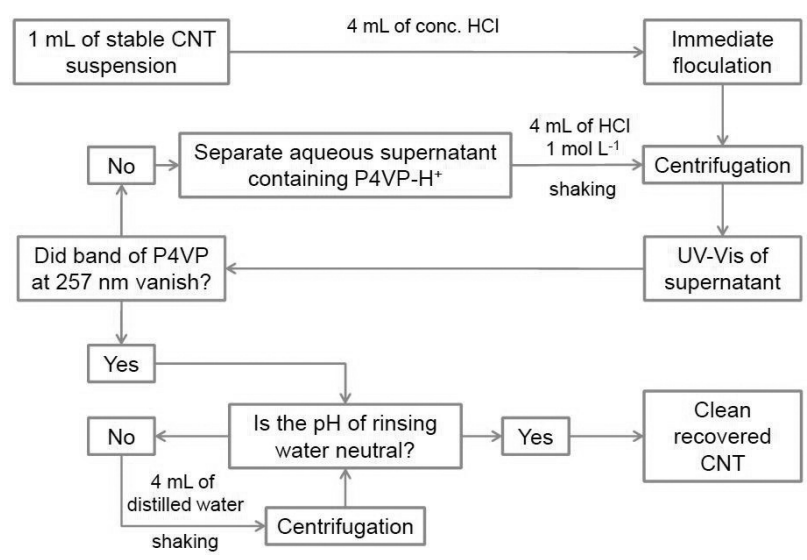

Figure 1. Scheme of the cyclic procedure followed to recover MWCNT after suspension assisted by P4VP.
Films for conductivity measurements

MWCNT/P4VP and MWCNT/metallopolymer films were prepared in the following manner: MWCNT (25 mg) was added to P4VP ethanolic solution (10 $\mathrm{mg} \mathrm{mL}^{-1}, 5 \mathrm{~mL}$ ) and the solution was ultrasonicated for $1 \mathrm{~h}$. After that, the solution was centrifuged at $4500 \mathrm{rpm}$ for 10 minutes and the supernatant dispersion was separated to be used in the film preparation. In addition, MWCNT (25 mg) was added to metallopolymer solution $\left(21.25 \mathrm{mg} \mathrm{mL}^{-1}, 5 \mathrm{~mL}\right)$ and the process was followed in the same way as described above in order to obtain the MWCNT/metallopolymer dispersion. These dispersions were then dropped onto the Teflon substrates, and dried by supplying a subtle flow of nitrogen at room temperature. This process was repeated until the films occupied the total volume $(15 \mathrm{~mm}$ of diameter, $2.2 \mathrm{~mm}$ of thickness). Finally, the films were dried in a vacuum oven under vacuum at $40{ }^{\circ} \mathrm{C}$ for 4 hours. The films could then be peeled off from the Teflon substrates to give samples with $2.2 \mathrm{~mm}$ of thickness.

\section{Results and Discussion}

\section{Interaction between P4VP and MWCNT}

Non-covalent interactions of organic molecules and carbon nanotubes can be mainly divided into $\pi$ - $\pi$ stacking, usually when condensed aromatic polycyclic compounds are used, ${ }^{14-16}$ or acid-base adduct formation where the nanotube behaves as an acid accepting electronic density of nitrogenated molecules in many cases. ${ }^{28-30}$ In order to check which interaction is dominant in P4VP/MWCNT pair, nuclear magnetic resonance experiments were conducted to investigate the effect of the presence of MWCNT on the chemical shifts of hydrogens and nitrogens of the polymer.

The ${ }^{1} \mathrm{H}$ and ${ }^{13} \mathrm{C}$ NMR spectra of the polymer in ethanol- $d_{6}$ presented the same features as reported in the literature $^{37}$ (see Supplementary Information section). The presence of MWCNT in suspension caused only a small broadening of the signals, not altering the overall pattern. Figure 2 shows the $\left[{ }^{1} \mathrm{H}^{15} \mathrm{~N}\right] \mathrm{HMBC}$ spectra of pure P4VP and the suspension containing P4VP/MWCNT. The nitrogen in P4VP appears at 299.44 ppm coupled with the ortho hydrogens (H2) at $8.28 \mathrm{ppm}$. When MWCNT is present, another signal at $299.07 \mathrm{ppm}$ for ${ }^{15} \mathrm{~N}$ and $8.22 \mathrm{ppm}$ for ${ }^{1} \mathrm{H}$ was observed. The presence of an extra signal in a more shielded position caused by the nanotube indicates that the pyridyl side chains are receiving electronic density upon interaction with carbon nanotube. This finding indicates that the whole ring is affected by the electron cloud of the nanotube. The nitrogens are also 

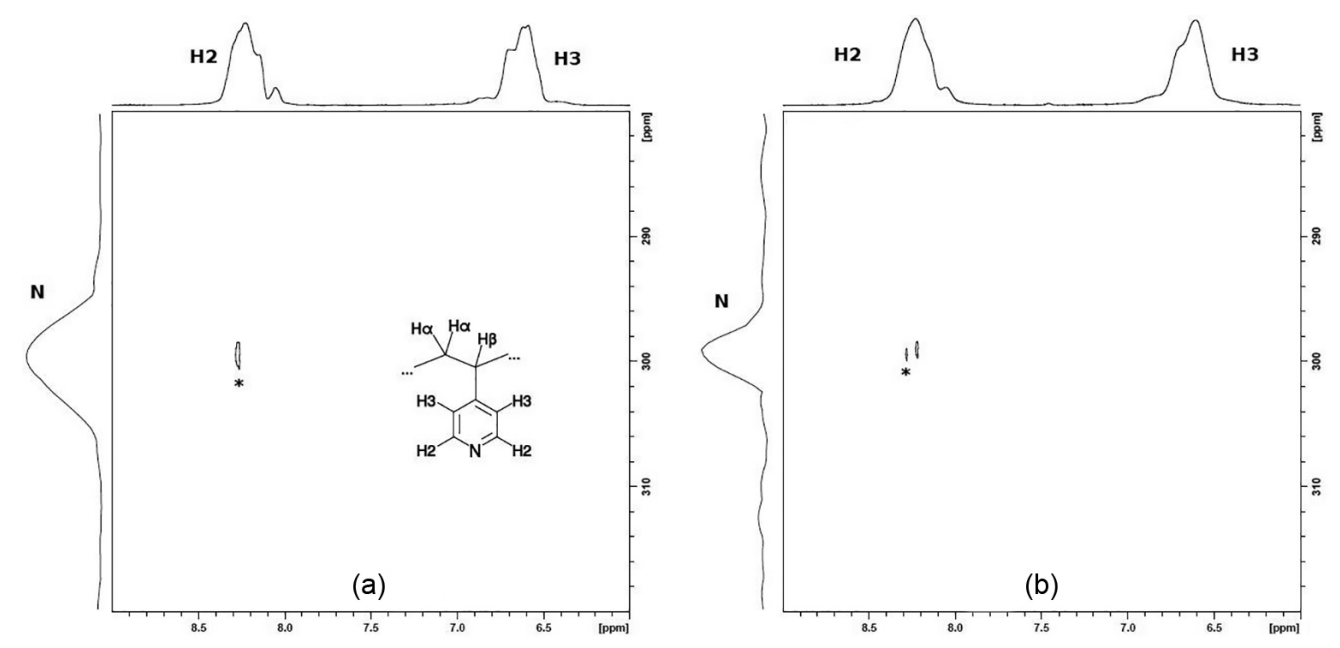

Figure 2. $\left[{ }^{1} \mathrm{H}^{-15} \mathrm{~N}\right] \mathrm{HMBC}$ spectra of pure P4VP (a) and the suspension containing P4VP and MWCNT (b) measured in ethanol- $d_{6}$. The $*$ indicates the signal of the pure P4VP.

shielded showing that, in the average, nitrogen does not donate electronic density to MWCNT walls and therefore charge-transfer interactions could be of minor importance. If charge-transfer was predominant, we would expect a deshielding of the nitrogen atom. Results from density functional theory of a single pyridine molecule adsorbed onto a graphene surface indicate that the van der Waals forces via $\pi-\pi$ stacking overcome the charge-transfer interaction via nitrogen lone pair. ${ }^{38}$

Interestingly, the suspension is not formed when P4VP is replaced by its isomer poly(2-vinylpyridine) ( $\mathrm{P} 2 \mathrm{VP}$ ), according to Rouse..$^{31} \mathrm{He}$ attributes the inefficiency of P2VP to the proximity of the polymer backbone to the nitrogen that hinders the charge-transfer interaction with nanotube walls. However, the reason could be that the P2VP-covered nanotubes are simply not well solvated and hence do not result in colloidal aggregates. The solubility of 4-vinylpyridine is $2.91 \mathrm{~g}$ per $100 \mathrm{~mL}$ of water whereas it is $2.75 \mathrm{~g}$ per $100 \mathrm{~mL}$ for 2 -vinylpyridine. ${ }^{39}$ These values suggest that the carbon backbone in the position 2 disfavors the solvation and may hamper the suspension formation.

\section{Determination of the mass of suspended MWCNT}

The formation of CNT suspension relies in a good balance between polymer-CNT and polymer-solvent interactions. For example, polyethyleneimine $\left(-\mathrm{CH}_{2} \mathrm{CH}_{2} \mathrm{NH}-\right)_{\mathrm{n}}$ (PEI) strongly $n$-dopes single-walled carbon nanotubes ${ }^{40}$ but is unable to suspend them in alcohols. Maybe due to the higher flexibility of PEI chain in comparison to P4VP, the PEI nitrogens strongly interact with the nanotube walls and few remain able to form hydrogen bond with the solvent to solvate the polymer-covered nanotube. ${ }^{31}$ Based on this information, we pursued a way to maximize polymer-solvent interactions in order to disrupt the colloidal aggregate and recover the MWCNT once suspended. Flocculation was achieved by adding concentrated $\mathrm{HCl}$ in the suspension, as shown in Figure S2. Protonation of the pyridyl groups may result in the solvation of pyridinium moieties by water and ethanol via ion-dipole interactions and disruption of $\pi-\pi$ stacking interactions with MWCNT wall, thus leading to flocculation.

MWCNTs previously suspended could be isolated after washing off the water-soluble poly(4-vinylpyridinium chloride). To assess the integrity of the MWCNTs after the procedure, they were analyzed by Raman spectroscopy. The spectra of recovered MWCNTs showed the same bands and similar relative intensity as before the interaction with the polymer, thus showing no structural modification upon sonication and acidic treatment. See Supplementary Information section for details.

The actual mass of suspended MWCNT and the composition of the mixtures are presented in Table 1. Pure P4VP and P4VP-Fe $(\mathrm{CN})_{5}{ }^{3-}$ metallopolymer were able to produce suspensions of up to 1.1 and $0.6 \mathrm{mg} \mathrm{mL}^{-1}$, respectively, which are concentrations above the reported range of $0.1-0.3 \mathrm{mg} \mathrm{mL}^{-1}$ used for high-throughput solution processing of carbon nanotubes. ${ }^{10,31,41,42}$ Comparing the suspended MWCNT mass of the samples P1, P2 and P3, one can note that the amount actually suspended is directly proportional to the amount of nanotubes mixed. However, the efficiency indicates that the suspended MWCNT mass in $\mathrm{P} 1$ relative to the initial mixed amount is less than in P3. This fact might be due to the higher viscosity of the highly-loaded sample, which hampers the suspension of a larger nanotube mass during the sonication time.

The metallopolymer samples were prepared using the mass of added MWCNT between P2 and P3, aiming the 
Table 1. Characteristics of suspensions of MWCNT in ethanol stabilized by P4VP-[Fe( $\left.(\mathrm{CN})_{5}\right]^{3-}$ metallopolymer

\begin{tabular}{|c|c|c|c|c|c|c|}
\hline Sample & $\mathrm{py}: \mathrm{Fe}^{\mathrm{a}}$ & $\begin{array}{l}\text { P4VP:MWCNT } \\
\text { (added) }(\mathrm{m} / \mathrm{m})\end{array}$ & $\begin{array}{l}\text { Added } \text { MWCNT }^{\mathrm{b}} / \\
\quad\left(\mathrm{mg} \mathrm{mL}^{-1}\right)\end{array}$ & $\begin{array}{c}\text { Suspended MWCNT } / \\
\left(\mathrm{mg} \mathrm{mL}^{-1}\right)\end{array}$ & Efficiency $^{\mathrm{c}} / \%$ & $\begin{array}{l}\text { P4VP:MWCNT } \\
\text { (suspended) }(\mathrm{m} / \mathrm{m})\end{array}$ \\
\hline P1 & - & $2: 1$ & 12.5 & 1.1 & 9 & $1: 0.044$ \\
\hline $\mathrm{P} 2$ & - & $2: 1$ & 5.0 & 0.9 & 18 & $1: 0.09$ \\
\hline P3 & - & $2: 1$ & 0.3 & 0.3 & 100 & $1: 0.5$ \\
\hline MP50.2 & $50: 1$ & $2: 1$ & 1.2 & 0.6 & 50 & $1: 0.25$ \\
\hline MP50.5 & $50: 1$ & $5: 1$ & 1.2 & 0.4 & 33 & $1: 0.17$ \\
\hline MP200.2 & $200: 1$ & $2: 1$ & 1.2 & 0.2 & 33 & $1: 0.08$ \\
\hline MP200.5 & $200: 1$ & $5: 1$ & 1.2 & 0.2 & 17 & 1:0.08 \\
\hline
\end{tabular}

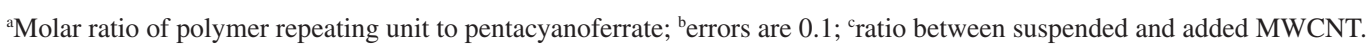

formation of a suspension with an appreciable mass of nanotubes. The actual amount of MWCNT suspended stayed between the values of $\mathrm{P} 2$ and $\mathrm{P} 3$, indicating that the presence of pentacyanoferrate bound to the polymer chain does not change drastically its ability to suspend nanotubes. We wondered if more free pyridyl groups in the metallopolymer chain would further increase the mass of suspend MWCNT and therefore we prepared samples using a metallopolymer having py:Fe ratio equals 200:1. However, the suspended content did not increase. The increase of the metallopolymer:MWCNT mass ratio from 2:1 to 5:1 (in samples MP50.5 and MP200.5) also did not suspend more nanotubes, in agreement with what was reported by Rouse. ${ }^{31}$

A possible explanation does not rely on the amount of free pyridyl groups but maybe on the conformation of the macromolecule in solution that exposes more the side chains for interaction with MWCNT. The gyration radius of a polyelectrolyte coil in low ionic strength medium is larger than a coil of neutral polymer with same molecular weight due to electrostatic repulsive forces. ${ }^{43}$ The evidence for that is the larger intrinsic viscosity of the metallopolymer bearing 50:1 monomer to pentacyanoferrate than the 200:1 reported previously. ${ }^{36}$ Therefore, the metallopolymer with more $\left[\mathrm{Fe}(\mathrm{CN})_{5} \text { py }\right]^{3-}$ in sample MP50.2 assumes a more extended conformation in solution than of sample MP200.2, making free pyridyl groups more available to interact with MWCNT. To corroborate this argument, the stability time of metallopolymer-stabilized suspensions was shorter ( 3 weeks) than the observed for suspensions with pure P4VP ( 8 weeks). The more elongated conformation of highly-charged metallopolymer chains wrapping MWCNT may allow a more rapid dissociation, favoring the aggregation kinetics. Added to this, the effect of the charge of the metallopolymer can contribute to a favorable interaction with solvent, destabilizing the suspension.

Another evidence rises when we compare the morphologies of dried nanocomposite films of MP50.2 and MP200.2 (Figures 3c and 3d, respectively). The former is more similar to the pure metallopolymer film (Figure 3b) than the latter, where the woven pattern of pristine MWCNT (Figure 3a) is more evident. This indicates that the nanotubes are better dispersed in the metallopolymer with higher pentacyanoferrate content (py:Fe ratio equals 50:1).
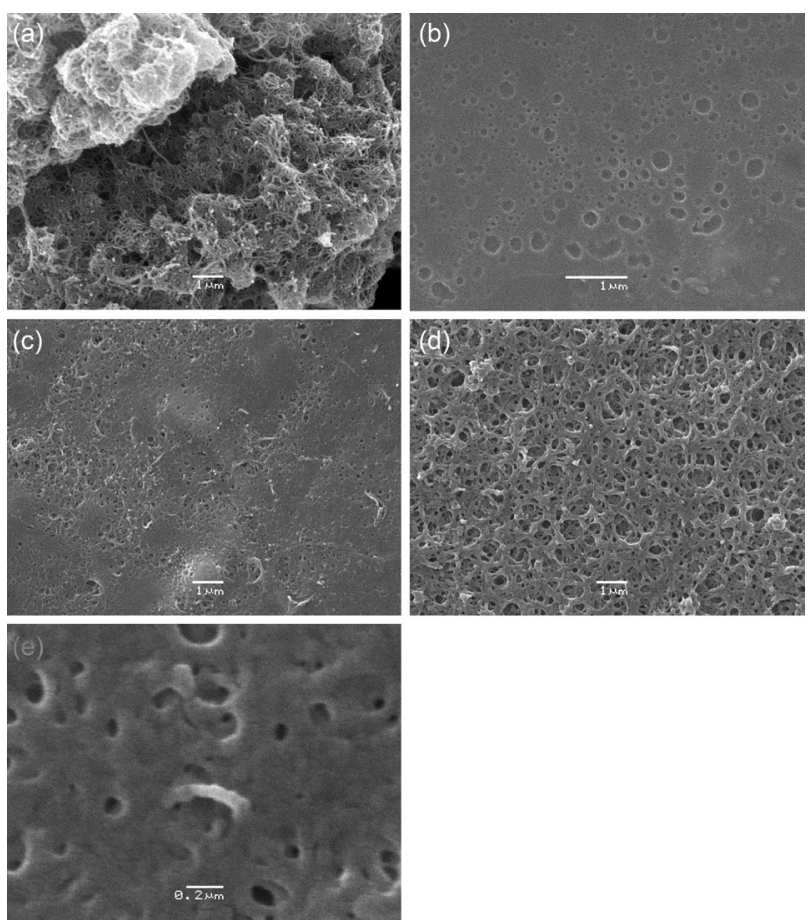

Figure 3. Scanning electron microscopy of (a) pristine MWCNT sonicated with ethanol; (b) metallopolymer with py:Fe ratio of 50:1; (c) the composite made with MWCNT and P4VP-Fe $(\mathrm{CN})_{5}$ metallopolymer with py:Fe = 50:1 (sample MP50.2); (d, e) the composite made with MWCNT and P4VP-Fe $(\mathrm{CN})_{5}$ with py: $\mathrm{Fe}=200: 1$ (sample MP200.2). MWCNT:P4VP weight ratio was 2:1 in (c-e). 
Interaction between $\left[\mathrm{Fe}(\mathrm{CN})_{5}\right]^{3-}$ and MWCNT

UV-Vis spectroscopy of the suspensions revealed the metal-to-ligand charge transfer (MLCT) band assigned as $\mathrm{Fe}(\mathrm{d} \pi) \rightarrow \mathrm{py}\left(\pi_{\mathrm{b} 1}^{*}\right),{ }^{44}$ which proves that $\left[\mathrm{Fe}(\mathrm{CN})_{5}\right]^{3-}$ are still coordinated to pyridyl moieties of the P4VP chain after interaction with MWCNTs. The position of this band of a model complex $\mathrm{Na}_{3}\left[\mathrm{Fe}(\mathrm{CN})_{5}\right.$ py], where py is pyridine, depends on solvent polarity. It is observed at $365 \mathrm{~nm}$ in water and at $400 \mathrm{~nm}$ in ethanol, as a consequence of the solvatochromic effect. . $^{45,46}$

The UV-Vis spectra of the P4VP-Fe(CN) metallopolymer with py:Fe ratio 200:1 in solvents composed by water and ethanol at different water molar fraction $\left(\mathrm{X}_{\mathrm{H}_{2} \mathrm{O}}\right)$ are presented in Figure 4. It can be observed that the MLCT band of the $\left[\mathrm{Fe}(\mathrm{CN})_{5}\right]^{3-}$ groups redshifts whereas the medium becomes water-poor, up to a maximum at $404 \mathrm{~nm}$, when the polymer chain starts to integrate the solvation sphere of pentacyanoferrates. ${ }^{36} \mathrm{When}$ the MWCNT is present, two bands appear in this region (solid line in Figure 4), a weak band at $360 \mathrm{~nm}$ indicating pentacyanoferrate immersed in a polar environment and another more intense band over $420 \mathrm{~nm}$, indicating that the pentacyanoferrates are located in a chemical environment even less polar than the solvent or the polymer chain. This evidence indicates proximity and interaction of the complexes with the hydrophobic MWCNT walls.

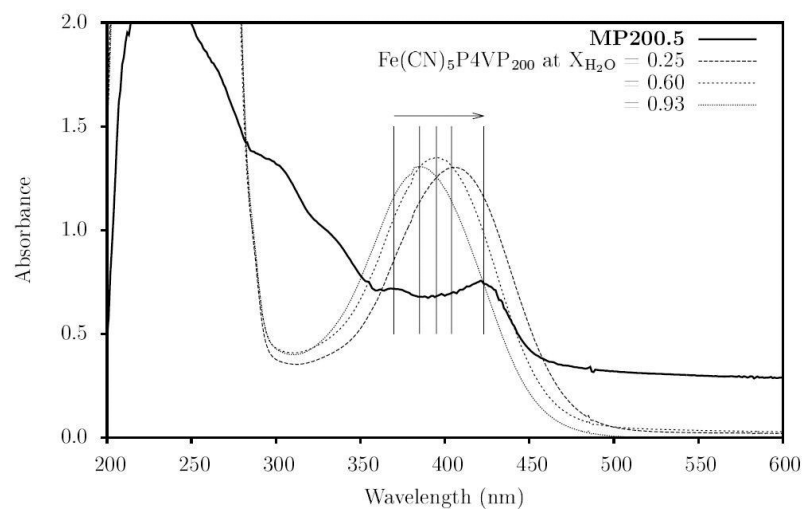

Figure 4. UV-Vis spectra of the MP200.5 sample and of the P4VP-Fe(CN) metallopolymer with py/Fe equals 200 at media composed by water and ethanol at different water molar fraction $\left(\mathrm{X}_{\mathrm{H}_{2} \mathrm{O}}\right)$. The vertical bars indicate the maximum of the MLCT band assigned to the $\left[\mathrm{Fe}(\mathrm{CN})_{5}\right]^{3-}$ groups. The arrow points to increasing hydrophobicity of the chemical environment of the complex.

\section{Characterization of the dried nanocomposite films}

Investigation using scanning electron microscopy of drop-cast samples revealed MWCNTs bundles in large agglomerates when no dispersing agent was added (Figure 3a). The films of metallopolymer samples with
py:Fe ratio of 50:1 and 200:1 were found to be very thin, having smooth and flat surfaces with round shapes only near the drop edge (Figure 3b) probably formed during the drying process. Inspection of the samples revealed that the good film-forming ability of P4VP is maintained when associated to MWCNT.

The nanocomposites formed with the metallopolymers are shown in Figures $3 \mathrm{c}$ and $3 \mathrm{~d}$. Linear features resembling MWCNT bundles are found embedded in polymeric matrix in both images. Nanotubes occur only together with the metallopolymer. The woven pattern found in Figure 3a is not found when they are dispersed with the metallopolymer, which is an indicative of good adhesion between both phases. Figure $3 e$ highlights in higher magnification a single bundle with diameter of $80 \mathrm{~nm}$ emerging from the underlying metallopolymer film. No clear interface between tubes and matrix is noticeable, suggesting good wetting and nice coverage.

Raman spectroscopy was used herein to investigate whether the decoration procedure maintain the structural integrity of MWCNT. It is known that the interaction between carbon nanotubes and an adsorbed species can be probed based on the shift of $\mathrm{G}$ band in single-walled $\mathrm{CNT},{ }^{47}$ but this information is not straightforward when dealing with multiwalled CNT. In composites of MWCNT, the majority of the walls scatters like a sample of pure MWCNT, since only the outermost wall interacts with the environment.

Raman spectra of MWCNT and dried metallopolymer/ MWCNT nanocomposites were normalized by the band at $1346 \mathrm{~cm}^{-1}$ and presented in Figure 5. The bands were fitted by lorentzian curves, whose parameters are shown in Table S1. The unscaled spectra in full range are depicted in Figure S5. The spectra of the metallopolymer/ MWCNT nanocomposites show the same features of the parent MWCNT, but small differences are noticeable after deconvolution. In the spectrum of MWCNT, the disorder-induced D band at $1346 \mathrm{~cm}^{-1}$ and its harmonic at $2690 \mathrm{~cm}^{-1}$ ( $\mathrm{G}^{\prime}$ band) are relatively intense when compared with tangential mode band $(\mathrm{G})$ at $1578 \mathrm{~cm}^{-1}$. High values of the intensity ratio $I_{D} / I_{G}$ between $D$ and $G$ bands are usually used as an indication of low quality graphitic material. ${ }^{48,49}$ In addition, the shoulder around $1613 \mathrm{~cm}^{-1}$ is typical of defective graphite-like materials and can be smaller in better quality MWCNT samples. ${ }^{50}$ After interaction with metallopolymers, the $\mathrm{I}_{\mathrm{D}} / \mathrm{I}_{\mathrm{G}}$ ratio calculated with intensity values after deconvolution decreased from 1.30 in the pure MWCNT to 1.01 and 1.05 in the MP50.2 and MP200.2 samples, respectively. This fact suggests that the polymeric chain interacts preferably with nanotubes with less defects on the outermost wall, to which they probably can better $\pi$-stack. Evaluating the $\mathrm{I}_{\mathrm{D}} / \mathrm{I}_{\mathrm{G}}$, or $\mathrm{I}_{\mathrm{G}} / \mathrm{I}_{\mathrm{G}}$, intensity ratios 

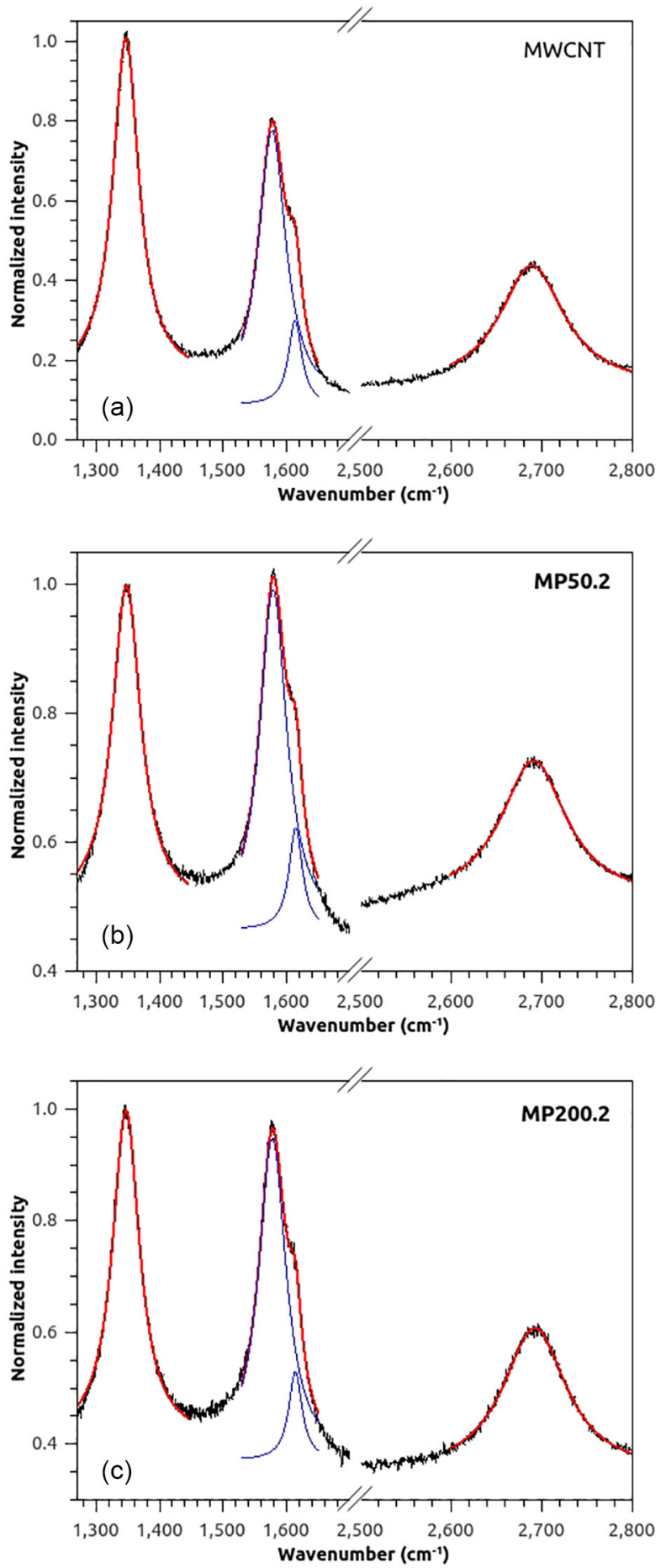

Figure 5. Normalized Raman spectra of pristine MWCNT (a) and the composites with MWCNT and P4VP-Fe $(\mathrm{CN})_{5}$ metallopolymer with py:Fe ratio $=50: 1$ (MP50.2 in b) and py:Fe = 200:1 (MP200.2 in c). The red lines are lorentzian fitting curves, whose details are found in the Supplementary Information section.

(Table S1) according to Maultzsch et al. ${ }^{51}$ the conclusion is the same. By the virtue of nanotubes being multiwalled, a clear evidence of nanotube-polymer interaction based of $\mathrm{G}$ band shift was not possible, as would be the case of SWCNT. ${ }^{47}$ Neither the signals of P4VP chain nor of $\left[\mathrm{Fe}(\mathrm{CN})_{5}\right]^{3-}$ groups were evident probably due to low scattering intensity.

Conductivity measurements showed ohmic behavior of both MWCNT/P4VP-Fe(CN) $)_{5}$ and MWCNT/P4VP films (see Supplementary Information section). The value of the conductivity were 2.35 and $0.95 \mathrm{mS} \mathrm{m}^{-1}$, respectively, whereas the pure P4VP is known to be an insulator, with conductivity value of $3.6 \mu \mathrm{S} \mathrm{m}^{-1} .52$ The presence of pentacyanoferrate did not change the semiconductor characteristic of the film, which is a property provided by dispersed MWCNTs. The enhanced conductivity of these nanocomposite films combined with the electrocatalytical properties of pentacyanoferrate have been explored to produce a superior electrochemical sensor for cysteine. ${ }^{34}$ Therefore, we hope that MWCNT/P4VP could serve as a versatile platform onto which different transition metal compounds can be anchored to produce solutionprocessable materials of diverse functionality.

\section{Conclusions}

Poly(4-vinylpyridine) and its metallopolymer bearing $\left[\mathrm{Fe}(\mathrm{CN})_{5}\right]^{3-}$ groups coordinated to pyridyl side chains are able to suspend up to 1.1 and $0.6 \mathrm{mg}$ of multiwalled carbon nanotube per $\mathrm{mL}$ of polar solvents such as ethanol and water/ethanol mixture upon sonication. Interactions of the type $\pi-\pi$ stacking between the aromatic side chains of poly(4-vinylpyridine) and the nanotube walls were pointed to be of the major relevance in the formation of suspensions in polar water/ethanol mixture upon sonication. Such interactions could be disrupted by addition of hydrochloric acid, allowing the measurement of the actual mass of dispersed nanotubes. Flocculated MWCNT could be isolated and presented no structural damage induced by the process, showing that their electronic and mechanical properties could be preserved. Variation of the py:Fe molar ratio in the metallopolymer and the P4VP:MWCNT weight ratio suggested that the amount of MWCNT suspended is not proportional to the amount of uncoordinated pyridyl groups. This fact might be related to the conformation of the macromolecule in solution when it interacts with the nanotubes. Finally, the system MWCNT/P4VP/Fe $(\mathrm{CN})_{5}{ }^{3-}$ consists in a supramolecular assembly gathering chemical species with very different polarity and properties. Under this perspective, a myriad of transition metal compounds could be coordinated to the P4VP/MWCNT aggregate in suspension and have their unique optical, magnetic, catalytic properties combined to the good film forming and conductive properties of the P4VP/MWCNT supramolecular platform. 


\section{Supplementary Information}

Supplementary information containing ${ }^{1} \mathrm{H}$ and ${ }^{13} \mathrm{C}$ NMR spectra of P4VP and P4VP/MWCNT; absorbance of P4VP band as function of washing cycles; Raman spectra of recovered MWCNT and current vs. potential data of fourprobes conductivity measurements of MWCNT/P4VP and MWCNT/P4VP/Fe $(\mathrm{CN})_{5}{ }^{3-}$ are available free of charge at http://jbcs.sbq.org.br as a PDF file.

\section{Acknowledgments}

We acknowledge financial support from São Paulo Research Foundation (FAPESP, grant 2010/09127-5), Conselho Nacional de Desenvolvimento Científico e Tecnológico (CNPq, grants 402627/ 2012-1, 202911/2014-4 and 203083/2015-6) and Coordenação de Aperfeiçoamento de Pessoal de Nível Superior (Capes) for a fellowship (LESCH). Raman experiments were performed using the facilities of the Laboratory of Advanced Optical Spectroscopy, LMEOA/IQ-UNICAMP (FAPESP 2009/54066-7). We thank Prof Marcelo Alexandre de Farias for help in the conductivity measurements and Eduardo Guimarães Ratier de Arruda for preparation of NMR samples. We thank Prof Maria Isabel Felisberti for helpful discussions and suggestions.

\section{References}

1. Saito, R.; Dresselhaus, G.; Dresselhaus, M. D.; Physical Properties of Carbon Nanotubes; Imperial College Press: London, UK, 1998.

2. Palacin, T.; Khanh, H. L.; Jousselme, B.; Jegou, P.; Filoramo, A.; Ehli, C.; Guldi, D. M.; Campidelli, S.; J. Am. Chem. Soc. 2009, 131, 15394

3. Zhao, H.; Zhu, Y.; Chen, C.; He, L.; Zheng, J.; Carbon 2012 , 50,4894

4. Campidelli, S.; Ballesteros, B.; Filoramo, A.; Daz, D. D.; de la Torre, G.; Torres, T.; Rahman, G. M. A.; Ehli, C.; Kiessling, D.; Werner, F.; J. Am. Chem. Soc. 2008, 130, 11503.

5. Ho, K. H. L.; Rivier, L.; Jousselme, B.; Jégou, P.; Filoramo, A.; Campidelli, S.; Chem. Commun. 2010, 46, 8731.

6. Wu, Z.-Y.; Huang, R.-B.; Xie, S.-Y.; Zheng, L.-S.; Dalton Trans. 2011, 40, 8353.

7. Stefopoulos, A. A.; Pefkianakis, E. K.; Papagelis, K.; Andreopoulou, A. K.; Kallitsis, J. K.; J. Polym. Sci., Part A: Polym. Chem. 2009, 47, 2551.

8. Winter, A.; Hager, M. D.; Newkome, G. R.; Schubert, U. S.; Adv. Mater. 2011, 23, 5728.

9. Jalali, F.; Hassanvand, Z.; Dorraji, P. S.; J. Braz. Chem. Soc. 2014, 25, 1537.
10. Banerjee, S.; Hemraj-Benny, T.; Wong, S.; Adv. Mater. 2005, 17, 17.

11. Balasubramanian, K.; Burghard, M.; Small 2005, 1, 180.

12. Tasis, D.; Tagmatarchis, N.; Georgakilas, V.; Prato, M.; Chem. Eur. J. 2003, 9, 4000.

13. Ajayan, P.; Tour, J.; Nature 2007, 447, 1066.

14. Yu, M.; Zu, S. Z.; Chen, Y.; Liu, Y. P.; Han, B. H.; Liu, Y.; Chem. Eur. J. 2010, 16, 1168.

15. Zhao, Y.-L.; Hu, L.; Gruener, G.; Stoddart, J. F.; J. Am. Chem. Soc. 2008, 130, 16996.

16. Zhao, Y. L.; Stoddart, J. F.; Acc. Chem. Res. 2009, 42, 1161.

17. Botelho, E. C.; Edwards, E. R.; Bittmann, B.; Burkhart, T.; J. Braz. Chem. Soc. 2011, 22, 2040.

18. Magadur, G.; Bouanis, F.; Norman, E.; Guillot, R.; Lauret, J.-S.; Huc, V.; Cojocaru, C.-S.; Mallah, T.; Chem. Commun. 2012, $48,9071$.

19. Stefano, J. S.; Montes, R. H.; Richter, E. M.; Muñoz, R. A.; J. Braz. Chem. Soc. 2014, 25, 484.

20. Etika, K.; Cox, M.; Grunlan, J.; Polymer 2010, 51, 1761.

21. Lee, J.-H.; Paik, U.; Choi, J.-Y.; Kim, K.; Yoon, S.-M.; Lee, J.; Kim, B.-K.; Kim, J.; Park, M.; Yang, C.; An, K.; Lee, Y.; J. Phys. Chem. C 2007, 111, 2477.

22. Wang, B.; Han, Y.; Song, K.; Zhang, T.; J. Nanosci. Nanotechnol. 2012, 12, 4664.

23. Singh, B.; Nayak, S.; Samal, S.; Bhattacharjee, S.; Besra, L.; J. Dispersion Sci. Technol. 2012, 33, 1021.

24. Bahun, G.; Wang, C.; Adronov, A.; J. Polym. Sci. Part A: Polym. Chem. 2006, 44, 1941.

25. le Goff, A.; Cosnier, S.; J. Mater. Chem. 2011, 21, 3910.

26. Yuan, W. Z.; Sun, J. Z.; Liu, J. Z.; Dong, Y.; Li, Z.; Xu, H. P.; Qin, A.; Häussler, M.; Jin, J. K.; Zheng, Q.; J. Phys. Chem. B 2008, 112, 8896 .

27. Lellouche, J. P.; Piran, M.; Shahar, L.; Grinblat, J.; Pirlot, C.; J. Mater. Chem. 2008, 18, 1093.

28. Lewenstein, J.; Burgin, T.; Ribayrol, A.; Nagahara, L.; Tsui, R.; Nano Lett. 2002, 2, 443.

29. Kong, J.; Franklin, N.; Zhou, C.; Chapline, M.; Peng, S.; Cho, K.; Dai, H.; Science 2000, 287, 622.

30. Chattopadhyay, D.; Galeska, I.; Papadimitrakopoulos, F.; J. Am. Chem. Soc. 2003, 125, 3370.

31. Rouse, J.; Langmuir 2005, 21, 1055.

32. Frehill, F.; Panhuis, M.; Young, N. A.; Henry, W.; Hjelm, J.; Vos, J. G.; J. Phys. Chem. B 2005, 109, 13205.

33. Parra-Alfambra, A.; Casero, E.; Ruiz, M.; Vázquez, L.; Pariente, F.; Lorenzo, E.; Anal. Bioanal. Chem. 2011, 401, 883.

34. Correa, C. C.; Jannuzzi, S. A. V.; Timm, R. A.; Santhiago, M.; Formiga, A. L. B.; Kubota, L. T.; Electrochim. Acta 2013, 113, 332.

35. Brauer, G.; Handbook of Preparative Inorganic Chemistry, vol. 1, $2^{\text {nd }}$ ed.; Academic Press: New York, USA, 1963. 
36. Jannuzzi, S. A. V.; Martins, B.; Felisberti, M. I.; Formiga, A. L. B.; J. Phys. Chem. B 2012, 116, 14933.

37. Pham, Q. T.; Pétiaud, R.; Watson, H.; Llauro-Darricades, M. F.; Proton and Carbon NMR Spectra of Polymers; Taylor \& Francis: New York, 1991.

38. Voloshina, E.; Mollenhauer, D.; Chiappisi, L.; Paulus, B.; Chem. Phys. Lett. 2011, 510, 220.

39. Scriven, E. F. V.; Murugan, R.; Kirk-Othmer Encyclopedia of Chemical Technology, vol. 20, ${ }^{\text {th }}$ ed.; Kroschwitz, J. I.; HoweGrant, M., eds; John Wiley \& Sons: Hoboken, 2005.

40. Shim, M.; Javey, A.; Shi Kam, N. W.; Dai, H.; J. Am. Chem. Soc. 2001, 123, 11512.

41. Vigolo, B.; Penicaud, A.; Coulon, C.; Sauder, C.; Pailler, R.; Journet, C.; Bernier, P.; Poulin, P.; Science 2000, 290, 1331.

42. Dan, B.; Irvin, G.; Pasquali, M.; ACS Nano 2009, 3, 835.

43. Jiang, L.; Yang, D.; Chen, S.; Macromolecules 2001, 34, 3730.

44. Formiga, A. L. B.; Vancoillie, S.; Pierloot, K.; Inorg. Chem. 2013, 52, 10653.

45. Toma, H.; Takasugi, M.; J. Solution Chem. 1983, 12, 547.
46. Macartney, D. L.; Rev. Inorg. Chem. 1988, 9, 101.

47. Dresselhaus, M.; Dresselhaus, G.; Saito, R.; Jorio, A.; Phys. Rep. 2005, 409, 47.

48. Knight, D. S.; White, W. B.; J. Mater. Res. 1989, 4, 385.

49. Zhang, L.; Li, H.; Yue, K.-T.; Zhang, S.-L.; Wu, X.; Zi, J.; Shi, Z.; Gu, Z.; Phys. Rev. B Condens. Matter Mater. Phys. 2002, $65,0734011$.

50. Jorio, A.; Pimenta, M.; Souza Filho, A.; Saito, R.; Dresselhaus, G.; Dresselhaus, M.; New J. Phys. 2003, 5, 139.

51. Maultzsch, J.; Reich, S.; Thomsen, C.; Webster, S.; Czerw, R.; Carroll, D.; Vieira, S.; Birkett, P.; Rego, C.; Appl. Phys. Lett. 2002, 81, 2647.

52. Chetia, J. R.; Moulick, M.; Dutta, A.; Indian J. Chem. Technol. 2004, 11, 80 .

Submitted: February 19, 2016

Published online: May 5, 2016

FAPESP has sponsored the publication of this article. 